\title{
Abstract: Articulated Head and Neck Patient Model for Adaptive Radiotherapy
}

\author{
Hendrik Teske ${ }^{1,2}$, Kathrin Bartelheimer ${ }^{1,2}$, Jan Meis ${ }^{1,2}$, Eva M. Stoiber ${ }^{1,2}$, \\ Rolf Bendl ${ }^{1,3}$, Kristina Giske ${ }^{1,2}$ \\ ${ }^{1}$ Division of Medical Physics in Radiation Oncology, DKFZ Heidelberg \\ ${ }^{2}$ National Center for Radiation Research in Oncology (NCRO), \\ Heidelberg Institute for Radiation Oncology (HIRO), Heidelberg \\ ${ }^{3}$ Faculty of Computer Science, Heilbronn University
}

h.teske@dkfz.de

State of the art radiotherapy enables for precise shaping of dose distributions to maintain tumor control while sparing organs at risk. Online adaptive radiotherapy improves that by taking into account inter-fractional changes of the anatomy throughout the treatment course. Especially variations in the posture of head and neck cancer patients cause large deformations in the anatomy, potentially leading to considerable deviations from the planned dose distributions. Adapting the treatment plan to compensate for such changes requires an accurate assessment of anatomical deformations. Commonly used models do not distinguish between bony tissue and soft tissue and thus result in unrealistic deformations of the bones. We propose an articulated patient model for the head and neck region, which inherently preserves the rigidity of the bones while allowing for modelling arbitrary postures of the patient. An articulated skeleton model is assembled from the segmentations of the bones in the planning CT. For an exemplary head and neck cancer patient, 40 individual bones were connected by 45 joints. All the joints were modelled as ball and socket joints, granting rotational mobility around the three body axes. Propagation of motion within the skeletal model is achieved by using an inverse kinematics approach [1]. On a consumer PC, different postures are generated interactively at a rate of $\sim 25$ per second. To investigate the advantages and limitations, this model was used to sample images of arbitrary postures of the patient. In addition to the skeletal model, a chainmail-based model was coupled to account for soft tissue deformation. Generation of realistic complex postures was achieved by using only up to 8 user-defined supporting vectors. The segmentations of the bones were generated manually. The resulting sampled images suggest that our model is able to accurately assess different inter-fractional postures of the patient. In combination with its time-efficient computation, it can further be used in the image registration context.

\section{References}

1. Sherman MA, Seth A, Delp SL. Simbody: multibody dynamics for biomedical research. Procedia IUTAM. 2011;2:241-61. 